\title{
ABC INEQUALITIES FOR SOME MODULI SPACES OF LOG-GENERAL TYPE
}

\author{
MinhyOng Kim
}

Let $B$ be a smooth projective curve of genus $\gamma$ over the complex numbers and let $f: A \rightarrow B$ be a non-isotrivial semi-abelian scheme over $B$ with projective generic fiber of relative dimension $g$. Let $U \subset B$ be the locus above which the fibers are projective, and let $S=B-U$ (a finite set). Thus $f: A_{U} \rightarrow U$ is abelian, and $f: A \rightarrow B$ is the connected component of its Neron model. Denote by $g_{0}$ the dimension of the fixed part of $A$ and $s=|S|$. We will adopt the convention of using the same notation for the map $f$ and several of its restrictions, unless an explicit danger of confusion forces us to do otherwise. Let $e: B \rightarrow A$ be the identity section, and let $W:=e^{*} \Omega_{A / B}$. Various authors have dealt with upper and lower bounds for the degree of $W$. Faltings [5], for example, shows that $\operatorname{deg}(W) \leq g(3 \gamma+s+1)$ while Moret-Bailly [7] shows that $\operatorname{deg}(W) \leq\left(g-g_{0}\right)(\gamma-1)$ in the case where $A / B$ is smooth. Arakelov [1] had earlier given the bound $\left(g-g_{0}\right)(\gamma-1+s / 2)$ when $A$ is the connected component of the Jacobian of a family of stable curves. In this paper, we improve a bit on Faltings, in the general case:

Theorem 1. Let $f: A \rightarrow B$ be a non-isotrivial semi-abelian scheme of relative dimension $g$ with projective generic fiber. Then

$$
\operatorname{deg}(W) \leq \frac{\left(g-g_{0}\right)}{2}(2 \gamma-2+s)
$$

where $g_{0}$ is the dimension of the fixed part and $s$ is the number of non-projective fibers.

Note that the degree is zero in the isotrivial case, so that the inequality still holds except for the obvious exception of $\gamma=0$.

The method of proof is an easy extension of Moret-Bailly's (and Szpiro's [10], which gives our general result for $g=1$ ), and is likely to be known to experts.

The reason it might still be worth writing down in full is because of the recently emerging connection with the $\mathrm{ABC}$ conjectures. That is, let $\mathcal{A}_{g, n}$ be the moduli space of principally polarized abelian varieties of dimension $g$ with full level- $n$ structure. For $n \geq 3$, we have that $\mathcal{A}_{g, n}$ is of log-general type. In fact, according to Mumford ([8], Proposition 3.4), $\mathcal{A}_{g, n}$ has a toroidal compactification $\mathcal{A}_{g, n} \hookrightarrow \overline{\mathcal{A}}_{g, n}$, such that the compactification divisor $D$ has normal crossings and

Received July 10, 1998. 
if $K$ is the canonical divisor of $\overline{\mathcal{A}}_{g, n}$, then $K+D$ is the pull-back of an ample line bundle on the Baily-Borel compactification $\mathcal{A}_{g, n}^{*}$. The geometric version of the $\mathrm{ABC}$ conjectures deals with maps from curves to varieties of log-general type [2]. In our case, if $P: B \rightarrow \overline{\mathcal{A}}_{g, n}$ is a map whose image does not lie in $D$, let $S=P^{-1}(D)$ (this is the inverse image as sets, without multiplicities) and $U=B-S$. Then there is a family $f: A \rightarrow B$ of semi-abelian varieties such that $A_{U}$ is the abelian family induced by $P \mid U$.(It also has a level structure which will be unimportant for our purposes.) As in the beginning, let $W=e^{*} \Omega_{A / B}$. Then $(\operatorname{det} W)^{(g+1)} \simeq P^{*}(K+D)([5]$, pp. 339-340). So as a result, we get

\section{Corollary 1.}

$$
\operatorname{deg}\left(P^{*}(K+D)\right) \leq \frac{g(g+1)}{2}(2 \gamma-2+s)
$$

This is an inequality of the sort conjectured by Buium for varieties of log general type, only more precise. Vojta has also conjectured such inequalities at the '98 ABC workshop in Tucson, AZ.

For completeness, we outline how one gets the same kind of inequalities for the moduli space of curves. That is, let $\mathcal{M}_{g, n}$ be the moduli space of curves of genus $g \geq 1$ with a level $n \geq 3$ structure on its Jacobian. Then $\mathcal{M}_{g, n}$ is of $\log$ general type ([8], Proposition 4.3). Let $\overline{\mathcal{M}}_{g, n}$ be the compactification of $\mathcal{M}_{g, n}$ constructed by taking the normalization in $\mathcal{M}_{g, n}$ of the Deligne-Mumford moduli space of stable curves. Finally, let $Y \rightarrow \overline{\mathcal{M}}_{g, n}$ be a smooth allowable modification ([8], p. 268), and $D \subset Y$ be the inverse image of the compactification divisor of $\overline{\mathcal{M}}_{g, n}$. Now, if $P: B \rightarrow Y$ is a map from a curve $B$ whose image does not lie in $D, S=P^{-1} D$, and $U=B-S$, then there is a family of stable curves $f: C \rightarrow B$ such that $C_{U}$ is the smooth family induced by $P \mid U$. Let $\omega_{C / B}$ be the relative dualizing sheaf. By Mumford ([8], proof of proposition 4.3), we have that $\operatorname{det}\left(f_{*}\left(\omega_{C / B}\right)\right)^{13}=P^{*}\left(K_{Y}+2 D\right)$. So we get the following corollary of Arakelov's inequality mentioned above:

$$
\operatorname{deg} P^{*}\left(K_{Y}+2 D\right) \leq \frac{13 g}{2}(2 \gamma-2+s) .
$$

\section{Proof of theorem}

Let $f: A \rightarrow B, U, S, s$ be as in the previous section. We need to prepare the situation a bit. First, let $A_{0}$ be the fixed-part. Then we may replace $A$ by the connected component of the Neron model of $A_{U} /\left(A_{0}\right)_{U}$, which is semi-stable since $A$ is, and use the fact that $e^{*}\left(\Omega_{A_{0} / B}\right)$ is trivial to reduce to the case where $A$ has no fixed-part (but the dimension is $g-g_{0}$ ).

Next, note that when we make a base-change that is unramified outside $S$, the quantities $2 \gamma-2+s$ and $\operatorname{deg}(W)$ just multiply by the degree of the base change. (The first by the Hurwitz formula, and the second by the fact that the formation of $W=e^{*}\left(\Omega_{A / B}\right)$ commutes with base change.) Thus, making a 
base-change to the field generated by the three-torsion of $A$ we may assume that the $A$ has a level three structure.

By Chai-Faltings [3], theorem VI.1.1, we have the existence of a proper smooth variety $X$ with a map $g: X \rightarrow B$ and an isomorphism $X_{U} \simeq A_{U}$, such that $D=g^{-1}(S)$ is a divisor with normal crossings and with the crucial property that

$$
g_{*}\left(\Omega_{X / B}(\log D)\right) \simeq W .
$$

On the other hand, associated to the family $f: A_{U} \rightarrow U$ we have the variation of complex Hodge structures $E_{0}:=R^{1} f_{*}\left(\Omega_{X_{U} / U}\right)$, which is (among other things) a bundle with a connection

$$
\nabla_{0}: E_{0} \rightarrow E_{0} \otimes \Omega_{U}
$$

$E_{0}$ also has the sub-bundle giving the Hodge filtration $F_{0} \subset E_{0}$ which we know to be canonically isomorphic to $W \mid U$. Denote by $E$ the Deligne extension of $E_{0}$ to a bundle on $B$ with a log-connection

$$
\nabla: E \rightarrow E \otimes \Omega_{B}(S) .
$$

Then by Steenbrink [9], $E$ has a sub-bundle $F$, the saturation of $F_{0}$, which can be identified with $g_{*}\left(\Omega_{X / B}(\log D)\right)$. Thus, we get an isomorphism $W \simeq F \subset E$. Hence, we need only bound the degree of $F$. Denote by $G_{0}$ the quotient $E_{0} / F_{0}$ which therefore extends to the bundle $G=E / F=R^{1} g_{*}\left(\mathcal{O}_{X}\right)$. The (flat) polarization $\langle\cdot, \cdot\rangle$ makes the bundle with connection $\left(E_{0}, \nabla_{0}\right)$ self-dual, and $F_{0}$ maximal isotropic, so that there is also an induced duality between $F_{0}$ and $G_{0}$. We will denote the duality paring between $F_{0}$ and $G_{0}$ by the same brackets as the polarization. We need the following

Lemma 1. The bundle $E$ is also self-dual.

Proof. In fact, it is self-dual as a bundle with log-connection. To see this recall that $E$ is characterized by the fact that it has a $\log$-connection $\nabla$ which extends the connection on $E_{0}$ and such that the residue operators on the points of $S$ have eigenvalues in the interval $[0,1)$. Since the dual connection $\nabla^{v}$ on the dual bundle $E^{v}$ is defined by $\nabla^{v} \phi(e)=d \phi(e)-\phi(\nabla e)$, we see that the eigenvalues of the residues of $\nabla^{v}$ are the negative of those associated to $\nabla$. However, since the abelian variety $A_{U}$ has semi-stable reduction, the monodromy operator is unipotent, and thus, the eigenvalues of the residues are zero. Thus they are also zero for $\nabla^{v}$. But this implies that $\left(E^{v}, \nabla^{v}\right)$ is also a Deligne extension for $\left(\left(E_{0}\right)^{v},\left(\nabla_{0}\right)^{v}\right) \simeq\left(E_{0}, \nabla_{0}\right)$, so we have an isomorphism $(E, \nabla) \simeq\left(E^{v}, \nabla^{v}\right)$.

From this lemma and the fact that a saturated subsheaf is determined by its generic stalk, we get that $F$ and $G$ are also in duality.

On $X$ we have the exact sequence of sheaves

$$
0 \rightarrow g^{*} \Omega_{B}(S) \rightarrow \Omega_{X}(\log D) \rightarrow \Omega_{X / B}(\log D) \rightarrow 0
$$


from which we get the log Kodaira-Spencer map

$$
\rho: F \rightarrow G \otimes \Omega_{B}(S) .
$$

As in [7], consider the exact sequences

$$
0 \rightarrow \Omega_{B}(S) \rightarrow g_{*}\left(\Omega_{X}(\log D)\right) \rightarrow N \rightarrow 0
$$

and

$$
0 \rightarrow N \rightarrow F \rightarrow G \otimes \Omega_{B}(S) .
$$

(Where $N$ is defined by these sequences.)

Lemma 2. $\operatorname{deg}(N)=0$.

Proof. We will prove this by showing that $N$ is preserved by the log-connection of $E$. This together with the fact that the eigenvalues of the residues for the logconnection are zero, will imply that $\operatorname{det} N$ has a regular connection, and hence, is of degree zero.

It suffices to check that $N \mid U \subset F_{0} \subset E_{0}$ is preserved by the connection, or even just to show that it is generically preserved by the connection. For this reason, we will be localizing on the base several times in the argument to follow, without special mention. For a section $\alpha$ of $\Omega_{X}$, denote by $[a]$ its image in $\Omega_{X / B}$.

Recall the usual computation of the Gauss-Manin connection [6] on the subspace $F_{0}$ of $E_{0}$ (We will do the computation on a section over $U$, the local sheaf theoretic argument being exactly the same.): Let $\phi \in F_{0}(U)$ so that it is an element of $\Omega_{X_{U} / U}\left(X_{U}\right)$. There exists a covering $\left\{U_{i}\right\}$ of $X_{U}$ such that $\phi$ is locally liftable to $\phi_{i} \in \Omega_{X_{U}}$. Let $t$ be a local parameter on the base and $v=\frac{d}{d t}$. Then $\nabla_{v} \phi$ has two components, the $F_{0}$ component is given locally by the formula $\left(\left(\nabla_{v} \phi\right)_{0}\right)_{i}=\left[\alpha_{i}\right] \in \Omega_{X_{U} / U}$, where

$$
d \phi_{i}=\alpha_{i} \wedge d t \in \Omega_{X}^{2},
$$

(From the fact that $[d \phi]=0$ in $\Omega_{X_{U} / U}^{2}$, we get that $d \phi_{i}$ is of the form $(\cdot) \wedge d t$, and the $\left[\alpha_{i}\right]$ 's glue together, although the $\alpha_{i}$ may not, in general) while the $G_{0}$ component is given by the 1-cocycle $\left\{\phi_{i}(v)-\phi_{j}(v)\right\}$. Now, when $\phi \in N$, from the first exact sequence defining $N$, there is a global (along the fibers) lifting $\phi^{\prime}$ of $\phi$ (after possibly shrinking $U$ ), and the $G_{0}$ component is zero, so we have the global element

$$
d \phi^{\prime} \in \Omega_{X}^{2}
$$

But we also have the contraction operator $i(v): \Omega_{X}^{2} \rightarrow \Omega_{X}^{1}$, so that we have an element $i(v)\left(d \phi^{\prime}\right) \in \Omega_{X_{U}}$. Locally, if we write $\left(d \phi^{\prime}\right)_{i}=\alpha_{i} \wedge d t$, we get that $i(v)\left(d \phi^{\prime}\right)=\alpha_{i}(v) d t-\alpha_{i}$, and hence, $\left[-i(v)\left(d \phi^{\prime}\right)\right]=\nabla_{v} \phi$, i.e., $\nabla_{v} \phi$ is globally liftable. So $\nabla_{v} \phi \in N$, as was to be shown.

Now we examine the map $\rho: F \rightarrow G \otimes \Omega_{B}(S)$. 
Lemma 3. $\rho$ factors through the dual of $F / N$ :

$$
\rho: F \rightarrow(F / N)^{v} \otimes \Omega_{B}(S) \subset G \otimes \Omega_{B}(S)
$$

Proof. Given the duality between $F$ and $G$, the dual of $F / N$ is exactly the annihilator in $G$ of $N \subset F$. Since all the subsheaves in question are saturated, we may check generically that the image of $\rho$ annihilates $N$. But $\rho$ relates to the Gauss-Manin connection according to the composition:

$$
\rho: F \subset E \stackrel{\nabla}{\rightarrow} E \otimes \Omega_{B}(S) \rightarrow G \otimes \Omega_{B}(S) .
$$

Now, if $s, t \in F_{0}$, we have $\langle s, t\rangle=0$ so for any local vector field $v$ on $U$,

$$
<\nabla_{v} s, t>+<s, \nabla_{v} t>=v<s, t>=0 .
$$

Now, the polarization induces the perfect pairing between $F_{0}$ and $G_{0}$ (by the isotropy of $F_{0}$ ). So we have

$$
<\rho(s), t>+<s, \rho(t)>=0
$$

for any $s, t \in F_{0}$. Hence, if we assume $s \in N$, then $\rho(s)=0$, so $<s, \rho(t)>=0$ for all $t \in F_{0}$, which is what we want.

From the lemma, we have an injection

$$
F / N \hookrightarrow(F / N)^{v} \otimes \Omega_{B}(S)
$$

of vector bundles of the same rank. Taking top exterior powers gives us an injection of line bundles, so

$$
\operatorname{deg}(F / N) \leq \operatorname{deg}(F / N)^{v}+r(2 \gamma-2+s),
$$

where $r$ is the rank of $F / N$, and hence,

$$
\operatorname{deg}(F / N) \leq \frac{r}{2}(2 \gamma-2+s) \leq \frac{\left(g-g_{0}\right)}{2}(2 \gamma-2+s)
$$

Since $N$ has degree 0 , we get

$$
\operatorname{deg}(W)=\operatorname{deg}(F) \leq \frac{\left(g-g_{0}\right)}{2}(2 \gamma-2+s)
$$

as was to be shown.

\section{Acknowledgement}

The author is grateful to Steve Zucker for discussions on Hodge theory related to this paper, in particular, for clearing up some confusion in the initial proof of Lemma 1. 


\section{References}

1. S. Arakelov Families of curves with fixed degeneracy, Izv. Akad. Nauk. 35 (1971), 12691293.

2. A. Buium The ABC theorem for abelian varieties, Inter. Math. Res. Not. 5 (1994), 220233.

3. C.-L. Chai and G. Faltings Degeneration of abelian varieties, Springer Verlag, BerlinHeidelberg-New York (1990).

4. P. Deligne Théorie de Hodge II, Publ. Math. IHES 40 (1971), 5-58.

5. G. Faltings Arakelov's theorem for abelian varieties, Invent. Math. 73 (1983), 337-347.

6. N. Katz Nilpotent connections and the monodromy theorem; application of a result of Turrittin, Publ. Math. I.H.E.S. 39 (1970), 355-232.

7. L. Moret-Bailly Pinceaux de variétés abélian, Astérisque 129, Société Mathématique de France, Paris (1985).

8. D. Mumford Hirzebruch's proportionality theorem in the non-compact case, Invent. Math. 42 (1977), 239-272.

9. J. Steenbrink Limits of Hodge structure, Invent. Math. 31 (1976), 229-257.

10. L. Szpiro Discriminant et conducteur des courbes elliptiques, in Séminaire sur les pinceaux de courbes elliptiques, Astérisque 183, Société Mathématique de France, Paris (1990).

Department of Mathematics, University of Arizona, Tucson, AZ 85721

E-mail address: kim@math.arizona.edu 